Volume 141, Number 12, December 2013, Pages 4103-4117

S 0002-9939(2013)12027-7

Article electronically published on August 7, 2013

\title{
GENERIC INCARNATIONS OF QUADRATIC TRANSFORMS
}

\author{
SHREERAM S. ABHYANKAR
}

(Communicated by Lev Borisov)

\begin{abstract}
The generic incarnation of a quadratic transform is obtained by adjoining an indeterminate. This is applied to proving some useful facts about intersection, such as the commutativity of contact numbers and the mixed characteristic generalization of Max Noether's Theorem on Refined Bezout.
\end{abstract}

\section{INTRODUCTION}

Given any two dimensional regular local domain $R$ whose characteristic is allowed to be different from the characteristic of its residue field $R / M(R)$, let $Q(R)$ be the set of all iterated quadratic transforms of $R$; i.e., $Q(R)$ is the set of all two dimensional regular local domains which birationally dominate $R$, i.e., dominate $R$ and have the same quotient field as the quotient field $L$ of $R$. By [Ab1] and $\mathrm{Ab2}$ we see that $Q(R)$ coincides with the disjoint union of $Q_{j}(R)$ taken over $j \in \mathbb{N}=$ the set of all nonnegative integers, where $Q_{j}(R)$ denotes the set of all $j$-th QDTs = Quadratic Transforms of $R$. Consider the rational function field $L(t)$ in an indeterminate $t$ over $L$. For every subring $S$ of $L$ let $S^{t}$ be the localization of $S[t]$ at the multiplicative set of all those members of $S[t]$ whose coefficients generate the unit ideal in $S$. Let $Q^{t}(R)=\left\{S^{t}: S \in Q(R)\right\}$. In our previous papers Ab13 and Ab14, of which this is a supplementary paper, we have shown that $Q^{t}(R) \subset Q\left(R^{t}\right)$; we may refer to $Q^{t}(R)$ as the generic incarnation of $Q(R)$. In Section 3 of this paper we shall use $Q^{t}(R)$ for proving the mixed characteristic generalization of Max Noether's Theorem on Refined Bezout, and then in Section 4 we shall use this to prove some results on contact numbers of complete ideals defined in Ab12 and AH3. In Section 5 we shall elucidate the multiplicity sequences of QDTs discussed in AL2. For any prime divisors $V$ and $V^{*}$ of $R$, in Theorems (4.3) and (4.4) of Section 4 we shall show how these sequences produce explicit formulas to calculate the $\left(V^{*}\right)$-value of the Zariski simple ideal $\zeta_{R}(V)$ belonging to $V$. Taking $V^{*}$ equals $V$ or ord ${ }_{R}$, we get formulas for $V\left(\zeta_{R}(V)\right)$ and $\operatorname{ord}_{R}\left(\zeta_{R}(V)\right)$ respectively.

Received by the editors February 1, 2012.

2010 Mathematics Subject Classification. Primary 14A05.

Key words and phrases. Contact number, generic incarnation, mixed characteristic. 


\section{TERMinOLOGY}

We shall mainly use the terminology of our papers Ab11 to Ab14. More background material can be found in our books and papers [Ab1] to Ab10, as well as in our joint papers AA1, AH1, AH2, AH3, AL1, and AL2 with Artal, Heinzer, and Luengo. Here are a few more terms which we shall use.

Recall that the length of a module $M$ over a ring $D$ is denoted by $\ell_{D} M$. Note that stating that $M$ is a finite $D$-module means that $M$ is finitely generated as a module.

By $\iota\left(\mathfrak{a}, \mathfrak{a}^{\prime} ; R\right)$ we denote the intersection multiplicity of any nonzero principal ideals $\mathfrak{a}, \mathfrak{a}^{\prime}$ in a two dimensional regular local domain $R$. For respective generators $a, a^{\prime}$ of $\mathfrak{a}, \mathfrak{a}^{\prime}$, we may write $\iota\left(a, a^{\prime} ; R\right)$ instead of $\iota\left(a R, a^{\prime} R ; R\right)$. Recall that

$$
\iota\left(a, a^{\prime} ; R\right)=\ell_{R}\left(R /\left(a, a^{\prime}\right) R\right)
$$

and note that this is zero or a positive integer or infinity according as the ideal $\left(a, a^{\prime}\right) R$ is the unit ideal or an $M(R)$-primary ideal or is contained in a nonzero nonunit principal ideal.

Let $t, t^{*}$ be independent indeterminates over a field $L$. Referring to the beginning of Section 2 of $\mathrm{Ab} 13$ ] for the definition of the $t$-extension $R^{t}$ of any subring $R$ of $L$, we recall that $R^{t}$ is the localization of the polynomial ring $R[t]$ at the multiplicative set of all polynomials $f(t) \in R[t]$ whose coefficients generate the unit ideal in $R$. We define the $\left(t, t^{*}\right)$-extension $R^{t, t^{*}}$ of $R$ by putting $R^{t, t^{*}}=\left(R^{t}\right)^{t^{*}}$. The ring $R^{t, t^{*}}$ can be directly defined as the localization of $R\left[t, t^{*}\right]$ at the multiplicative set of all polynomials $f\left(t, t^{*}\right) \in R\left[t, t^{*}\right]$ whose coefficients generate the unit ideal in $R$. Note that if $R$ is a regular local domain of dimension $d$ with quotient field $L$, then $R^{t}$ and $R^{t, t^{*}}$ are regular local domains of dimension $d$ with quotient fields $L^{t}=L(t)$ and $L^{t, t^{*}}=L\left(t, t^{*}\right)$ respectively.

For any ring $A$ we put

$$
P(A)=\text { set of all nonzero principal ideals in } A
$$

with

$$
P^{\times}(A)=P(A) \backslash\{A\} \text { and } P^{*}(A)=P(A) \cap \operatorname{spec}(A) .
$$

For any overring $B$ of $A$ we put

$$
\bar{A}^{B}=\text { integral closure of } A \text { in } B
$$

and we note that $\bar{A}^{B}$ is a subring of $B$, and for any ideal $J$ in $A$ we put

$$
J^{-B}=\text { the integral closure of } J \text { in } B
$$

and we note that $J^{-B}$ is an ideal in $\bar{A}^{B}$.

If $B$ is quasilocal, then $H_{B}: B \rightarrow H(B)=B / M(B)$ denotes the residue class epimorphism, and then put

$$
\chi(A, B)=\left[K^{\prime}: K\right],
$$

where $K^{\prime}$ is the algebraic closure of $K=\mathrm{QF}\left(H_{B}(A)\right)$ in $H(B)$.

Recall that for any DVR $V$ and any $a \in \mathrm{QF}(V)$ we put $V(a)=\operatorname{ord}_{V} a$, and for any ideal $I$ in a noetherian subring $S$ of $V$ we put

$$
V(I)=\operatorname{ord}_{V} I=\min \{V(a): a \in I\}
$$

with the understanding that $V(I)=\infty$ if $I=\{0\}$. 
In Proposition (3.5) of [Ab12, for any $V$ and $V^{*}$ in $D(R)^{\times}=$set of all prime divisors of a two dimensional regular local domain $R$ with quotient field $L$, the contact number $c\left(R, V, V^{*}\right)$ of $V$ with $V^{*}$ at $R$ was defined by putting

$$
c\left(R, V, V^{*}\right)=\operatorname{ord}_{V} \zeta_{R}\left(V^{*}\right)
$$

where we have the Zariski map $\zeta_{R}: D(R)^{\Delta} \rightarrow C(R)=$ set of all $M(R)$-primary simple complete ideals in $R$.

To generalize the contact number, let $I$ and $I^{*}$ be any complete $M(R)$-primary ideals in $R$. By the Zariski Factorization Theorem proved in Appendix 5 of volume II of [ $\mathrm{Zar}$ and restated in (2.4) of [Ab12, we can uniquely write

$$
I=\prod_{1 \leq i \leq h} \zeta_{R}\left(V_{i}\right)^{n(i)} \quad \text { with } \quad n(i) \in \mathbb{N}_{+}
$$

where $V_{1}, \ldots, V_{h}$ are pairwise distinct members of $D(R)^{\Delta}$ with $h \in \mathbb{N}_{+}$, and

$$
I^{*}=\prod_{1 \leq j \leq h^{*}} \zeta_{R}\left(V_{j}^{*}\right)^{n^{*}(j)} \quad \text { with } \quad n^{*}(j) \in \mathbb{N}_{+}
$$

where $V_{1}^{*}, \ldots, V_{h^{*}}$ are pairwise distinct members of $D(R)^{\Delta}$ with $h^{*} \in \mathbb{N}_{+}$. We define the contact number $c\left(R, I, I^{*}\right)$ of $I$ with $I^{*}$ at $R$ by putting

$$
c\left(R, I, I^{*}\right)=\sum_{1 \leq i \leq h, 1 \leq j \leq h^{*}} n(i) n^{*}(j) \chi\left(R, V_{i}\right) c\left(R, V_{i}, V_{j}^{*}\right),
$$

and we note that this is always a positive integer.

\section{Refined Bezout}

Let $R$ be a two dimensional regular local domain with quotient field $L$. For any $T \in Q(R)$ and any nonzero ideal $I$ in $R$, in Section 2 of Ab11 we have defined the $(R, T)$-transform of $I$ as a nonzero ideal $(R, T)(I)$ in $T$, and in Section 2 of Ab13. we have defined the singularity tree $\mathfrak{P}(R, F)$ of any $0 \neq F \in R$ by putting

$$
\mathfrak{P}(R, F)=\{T \in Q(R):(R, T)(F R) \neq T\}
$$

and its $j$-th layer by putting $\mathfrak{P}_{j}(R, F)=\mathfrak{P}(R, F) \cap Q_{j}(R)$. In this section we shall prove:

Refined local Bezout Theorem (3.1). Let $F, G$ be nonzero elements in $M(R)$ such that the ideal $(F, G) R$ is $M(R)$-primary. Then we have

$$
\iota(F, G ; R)=\sum_{T \in Q(R)} \chi(R, T) d_{T} e_{T}
$$

with $d_{T}=\operatorname{ord}_{T}(R, T)(F T)$ and $e_{T}=\operatorname{ord}_{T}(R, T)(G T)$, where the sum is essentially finite because $d_{T} e_{T}=0$ for all $T \notin \mathfrak{P}(R, F) \cap \mathfrak{P}(R, G)$ and $\mathfrak{P}(R, F) \cap \mathfrak{P}(R, G)$ is a finite set.

Note 3.1.1. It seems likely that in the classical cases when $R$ is the local ring of a point in the plane over the complex field $\mathbb{C}$, the idea of this theorem originated in the writings of Max Noether; see pages 146-148 of [Ab6]. We shall divide the proof of (3.1) into several lemmas. Recall that $F, G$ are nonzero elements in $M(R)$ such that the ideal $(F, G) R$ is $M(R)$-primary. Henceforth let $d=\operatorname{ord}_{R} F$ and $e=\operatorname{ord}_{R} G$. 
Notation 3.1.2. Referring to pages 206-209 of [Ab8 for the definition of graded rings and homogeneous ideals, consider the naturally graded ring

$$
\operatorname{grad}(R)=A=\sum_{i \in \mathbb{N}} A_{i} \text { with } A_{i}=M(R)^{i} / M(R)^{i+1},
$$

and let us put

$$
\bar{P}(A)=\text { set of all nonzero homogeneous principal ideals in } A
$$

with

$$
\bar{P}^{\times}(A)=\bar{P}(A) \backslash\{A\} \text { and } \bar{P}^{*}(A)=\bar{P}(A) \cap \operatorname{spec}(A) .
$$

Given any generators $(x, y)$ of $M(R)$, as in Remark (4.0) of Ab11, we may identify $A$ with the polynomial ring $K[X, Y]$ in indeterminates $X, Y$ over the field $K=$ $H(R)$. Note that now $\operatorname{info}(F)$ is a nonzero homogeneous polynomial of degree $d$ in $X, Y$ with coefficients in $K$. As in (4A) of Section 4 of [AA1, we have a bijection

$$
\delta_{R}: \bar{P}^{*}(A) \rightarrow Q_{1}(R)
$$

which is called the tangential bijection relative to $R$, and for any $\Psi \in \bar{P}^{*}(A)$, $\delta_{R}(\Psi)$ is called the QDT of $R$ in the direction $\Psi$. For any $\Phi \in \bar{P}(A)$ put

$$
\delta_{R}^{\prime}(\Phi)=\left\{\delta_{R}(\Psi): \Psi \in \bar{P}^{*}(A) \text { with } \Phi \subset \Psi\right\},
$$

where we call $\delta_{R}^{\prime}$ the derived tangential map relative to $R$. By the GCD of any nonempty subset $N$ of nonzero homogeneous elements in $A$ we mean the smallest nonzero homogeneous principal ideal $\operatorname{GCD}(N)$ in $A$ such that $N \subset \operatorname{GCD}(N)$. For any nonzero ideal $I$ in $R$, upon letting $r=\operatorname{ord}_{R} I$ and taking $N$ to be the set of all the infos of elements of $I$ of $R$-order $r$, we put $\mathfrak{f}(R, I)=\operatorname{GCD}(N)$, and we call $\mathfrak{f}(R, I)$ the Zariski gcd of $(R, I)$. By $s(R, J)$ we denote the $(X, Y)$-degree of a generator of $\mathfrak{f}(R, I)$; we call $s(R, I)$ the suborder of $(R, I)$. The Zariski number of $(R, I)$ is defined by putting $m(R, I)=r-s(R, I)$, and for any $T \in Q(R)$ the derived Zariski number is defined by putting $m(R, I, T)=m(T,(R, T)(I))$. If $J$ is a nonzero subideal of $I$ with $\operatorname{ord}_{R} J=\operatorname{ord}_{R} I$, then clearly

$$
\mathfrak{f}(R, J) \subset \mathfrak{f}(R, I)
$$

and hence

$$
\mathfrak{f}(R, J)=\mathfrak{f}(R, I) \mathfrak{g}(R, I, J)
$$

for a unique nonzero homogeneous principal ideal $\mathfrak{g}(R, I, J)$ in $\operatorname{grad}(R)$. We call $\mathfrak{g}(R, I, J)$ the reduced gcd of $(R, I, J)$, we put

$$
\mathfrak{P}_{1}(R, I, J)=\delta_{R}^{\prime}(\mathfrak{g}(R, I, J)),
$$

and we call $\mathfrak{P}_{1}(R, I, J)$ the reduced first layer of $(R, I, J)$. Note that if the ideal $J$ is principal, then $\operatorname{info}(R, J)=\mathfrak{f}(R, J)$. Recall that the big stars of $I$ in $R$ are members of $\mathfrak{B}(R, I)=\left\{o_{R}^{-1}(V): V \in \mathfrak{D}(R, I)\right\}=\{T \in \mathfrak{Q}(R, I): m(R, I, T)>0\}$.

Note 3.1.3. It can easily be seen that the usual definition of a UFD (page 13 of Ab8) is equivalent to the definition of a UFB = Unique Factorization Box according to which a UFB is a domain $B$ for which there exists an injective map $E_{B}: W_{B} \rightarrow B^{\times}$from an indexing set $W_{B}$ such that every $b \in B^{\times}$can be expressed uniquely as

$$
b=u(b) \prod_{w \in W_{B}} E_{B}(w)^{N_{B}(b, w)} \text { with } u(b) \in U(B)=\text { set of all units in } B
$$


where $N_{B}(b, w) \in \mathbb{N}$ is such that, for any $b \in B^{\times}, N_{B}(b, w)=0$ for all except a finite number of $w$. Here box refers to an equivalence class of irreducible associates in $B^{\times}$. We have chosen one representative from each box and labelled these representatives as $E_{B}(w)_{w \in W_{B}}$. Note that

$$
w \mapsto E_{B}(w) B \text { gives a bijection } W_{B} \rightarrow P^{*}(B)=\operatorname{spec}_{1}(B)
$$

where $\operatorname{spec}_{1}$ denotes the set of all height-one members of spec.

Note 3.1.4. Let $(x, y)$ be generators of $M(R)$ and let $B=R[y / x]$.

Note that $M(R) B=x B \in P^{*}(B)$ and $B_{x B}=o(R)$; let us denote this DVR by $V$. Then any $p \in R^{\times}$can be expressed as $p=\Phi(x, y)$ where $\Phi(X, Y)$ is a homogeneous polynomial of degree $V(p)$ in indeterminates $X, Y$ with coefficients in $R$, at least one of which is not in $M(R)$. Upon letting $\Phi^{\prime}(Y)=\Phi(X, Y) / X^{V(p)}$ we get $\Phi^{\prime}(Y) \in R[Y]$, and hence upon letting $q(p)=\Phi^{\prime}(y / x)$ we obtain $q(p)=$ $p / x^{V(p)} \in B$. We extend the definition of $q$ by putting $q(p R)=q(p) B$. Note that $p R$ and $q(p R)$ belong to $P(R)$ and $P(B)$ respectively and that $q(p R)$ is independent of the particular generator $p$. Also note that $x R \in P^{*}(R)$, but $q(x R)$ is the unit ideal $B$. Finally note that

$$
\left\{\begin{array}{l}
\text { for any } t \neq w \text { in } P^{*}(R) \backslash\{x R\} \text { we have } \\
\operatorname{ord}_{R_{w}} q(w)=1 \neq 0=\operatorname{ord}_{R_{w}} t=\operatorname{ord}_{R_{w}} x \\
\text { and } \operatorname{ord}_{R_{t}} q(t)=1 \neq 0=\operatorname{ord}_{R_{t}} w=\operatorname{ord}_{R_{t}} x \\
\text { and hence } q(w) \neq q\left(w^{*}\right) .
\end{array}\right.
$$

Convert the UFD $R$ into a UFB by constructing an injective map

$$
E_{R}: W_{R}=P^{*}(R) \rightarrow R^{\times} \text {given by } E_{R}(w)=\text { a generator of } w
$$

and obtain an injective map

$$
E_{B}: E_{B}=P^{*}(R) \rightarrow B^{\times} \text {by putting } E_{B}(w)= \begin{cases}q(w) & \text { if } w \neq x R \\ x & \text { if } w=x R\end{cases}
$$

and note that for all $w \in P^{*}(R)$ we have

$$
V(w)=V\left(E_{R}(w)\right) \in \mathbb{N}_{+} \text {and } V\left(E_{B}(w)\right)= \begin{cases}0 & \text { if } w \neq x R \\ 1 & \text { if } w=x R .\end{cases}
$$

Any $b \in B^{\times}$can be written as $b=\Psi^{\prime}(y / x)$ with $\Psi^{\prime}(Y) \in R[Y]$ of some degree $c \in \mathbb{N}$, and upon letting $\Psi(X, Y)=X^{c} \Psi^{\prime}(Y / X)$ we see that $\Psi(X, Y)$ is a homogeneous polynomial of degree $c$ in $X, Y$ with coefficients in $R$, and hence $b x^{c}=\Psi(x, y) \in R$ with $\Psi(x, y) \in M(R)^{c}$. Thus

$$
\left\{\begin{array}{l}
\text { for every } b \in B^{\times} \text {there is } c^{\prime} \in \mathbb{N} \text { such that } \\
\text { for any } c \in \mathbb{N} \text { with } c \geq c^{\prime} \\
\text { upon letting } r=b x^{c} \text { we have } r \in M(R)^{c} .
\end{array}\right.
$$

Factoring $r$ in $R$ we get

$$
r=u(r) \prod_{w \in P^{*}(R)} E_{R}(w)^{N_{R}(r, w)} \text { with } u(r) \in U(R) \text { and } N_{R}(r, w)=\operatorname{ord}_{R_{w}} r \in \mathbb{N} .
$$


Comparing the $V$ values of the two sides of the above equation we get

$$
c+V(b)=\sum_{w \in P^{*}(R)} V\left(E_{R}(w)\right) N_{R}(r, w)
$$

and clearly $V(b) \in \mathbb{N}$, and hence upon letting

$$
N_{B}(b, w)= \begin{cases}N_{R}(r, w) & \text { if } w \neq x R \\ V(b) & \text { if } w=x R\end{cases}
$$

and dividing both sides of the above factorization of $r$ by $x^{c}$ we get the factorization in $B^{\times}$given by

(6)

$$
b=u(b) \prod_{w \in W_{B}} E_{B}(w)^{N_{B}(b, w)} \text { with } u(b)=u(r) \in U(R) \subset U(B) \text { and } N_{B}(b, w) \in \mathbb{N} .
$$

By (1) we see that the above factorization is unique. By (1) to (6) we see that the domain $B=R[y / x]$ has the following factorization properties:

(7) $B$ is a UFB and hence a UFD. We have $U(B)=U(R)$.

(8) $w \mapsto\left(E_{R}(w) / x^{V(w)}\right) B$ gives a bijection $P^{*}(R) \backslash\{x R\} \rightarrow P^{*}(B) \backslash\{x B\}$.

(9) $w^{\prime} \mapsto w^{\prime} \cap R$ gives the inverse of the bijection of $(2)$.

(10) For any $a \in R \backslash\{x R\}$ we have $(q(a) B) \cap R=a R$.

(11) For any $a \in R \backslash\{x R\}$ and $p \in R$ such that $(a, p) R$ is $M(R)$-primary, and any $b \in B$ such that $p b \in q(a) B$, we have $b \in q(a) B$.

Note 3.1.5. Let $(x, y)$ be generators of $M(R)$. Since $\operatorname{ord}_{R} F=d$, we can write

$$
F=f(x, y) \text { where } f(X, Y)=\sum_{0 \leq i \leq d} a_{i} x^{i} y^{d-i} \text { with } a_{i} \in R
$$

Let $B=R[y / x]$ and $F^{\prime}=F / x^{d}$. Then $F^{\prime}=f(1, y / x) \in B$. Similarly, upon letting $G^{\prime}=G / x^{e}$ we get $G^{\prime} \in B$. Now assume that $\operatorname{ord}_{(R / x R)} F=d$. This is clearly equivalent to saying that $\mathfrak{f}(R, F R) \not \subset X A$, and it implies $F \notin x R$ and hence $\left(F^{\prime} B\right) \cap R=F R$ by (3.1.4)(10). Let $\phi: B \rightarrow C=B / F^{\prime} B$ be the residue class ring epimorphism and let $S=\phi(R), g=\phi(G), g^{\prime}=\phi\left(G^{\prime}\right)$, and $z=\phi(y / x)$. We claim that:

(1) $C$ is a finite $S$-module with $\ell_{S}(C / S)<\infty$.

(2) $g$ is a nonzerodivisor on $C$. Note that $\zeta \in S$ is a nonzerodivisor on an $S$-module $D$ means that $\zeta \eta=0$ with $\eta \in D \Rightarrow \eta=0$.

(3) $z^{n}$ is a nonzerodivisor on $C$ for all $n \in \mathbb{N}$.

To prove (1), since $\mathfrak{f}(R, F R) \not \subset X A$, looking at the above displayed expression of $f(X, Y)$ we see that $a_{0} \notin M(R)$ and hence $z$ satisfies the monic polynomial equation $f^{*}(z)=0$ over $S$ where

$$
f^{*}(Y)=Y^{d}+\sum_{1 \leq i \leq d} \phi\left(a_{i} / a_{0}\right) Y^{d-i}
$$

Thus $z$ is integral over $S$. Therefore, since $C=S[z], \mathrm{L} 4 \S 10 \mathrm{E}(2)$ of Ab8] tells us that $C$ is a finite $S$-module. Consequently, by (3.1.4)(3) we see that $z^{n} C \subset S$ for some positive integer $n$. Since $F \notin x R$, we see that the ideal $z^{n} S$ is $M(S)$-primary. Hence, referring to $\mathrm{L} 4 \oint 5 \mathrm{O}(25)$, we conclude that $C / S$ is an artinian module over the artinian ring $S / z^{n} S$ and therefore $\ell_{S / z^{n} S}(C / S)<\infty$. Clearly $\ell_{S}(C / S)=$ $\ell_{S / z^{n} S}(C / S)$ and therefore $\ell_{S}(C / S)<\infty$. 
This proves (1). (2) follows by taking $(a, p)=(F, G)$ in $(3.1 .4)(11)$. (3) follows by taking $(a, p)=\left(F, x^{n}\right)$ in $(3.1 .4)(11)$.

Lemma 3.2. Assume that $M(R)$ has generators $x, y$ such that $\operatorname{ord}_{(R / x R)} F=d$ and $G \notin x R$. Let $B=R[y / x]$ with $F^{\prime}=F / x^{d}$ and $G^{\prime}=G / x^{e}$. Then $F^{\prime} \in B$ with $\left(F^{\prime} B\right) \cap R=F R$ and $G^{\prime} \in B$ with $\left(G^{\prime} B\right) \cap R=G R$ by the preamble of (3.1.5). Moreover, upon letting $\phi: B \rightarrow C=B / F^{\prime} B$ be the residue class ring epimorphism and upon letting $S=\phi(R)$ with $g=\phi(G)$ and $g^{\prime}=\phi\left(G^{\prime}\right)$ with $z=\phi(x)$, the $S$ modules $C / S, S / g S, C / g S, C / g^{\prime} C, C / z C, C / z^{e} C$ have finite $S$-lengths satisfying the following five equations:

(1) $\ell_{S}(C / g C)=\ell(S / g S)$,

(2) $\ell_{S}(C / g C)=\ell_{S}\left(C / z^{e} C\right)+\ell_{S}\left(C / g^{\prime} C\right)$,

(3) $\ell_{S}(C / S)=\ell_{S}(C / z C)=d$,

(4) $\ell_{S}\left(C / z^{e} C\right)=d e$,

(5) $\ell_{S}(S / g S)=d e+\ell_{S}\left(C / g^{\prime} C\right)$.

Proof. In the following proofs of (1) to (3) we shall use the isomorphism $C / D \approx$ $\eta C / \eta D$ of $S$-modules where $D$ is a submodule of $C$ and $\eta \in S$ is a nonzerodivisor on $C$. Moreover we shall tacitly use (3.1.5)(1) and some obvious facts on finiteness of length.

First: Looking at the diagram of $S$-modules below, we have the two ways of inserting a module between $g S \subset C$ exhibited by the left half $g S \subset S \subset C$ and the right half $g S \subset g C \subset C$. This yields the equation $\ell_{S}(C / S)+\ell_{S}(S / g S)=$ $\ell_{S}(C / g C)+\ell_{S}(g C / g S)$. We get the isomorphism $C / S \approx g C / g S$ because by $(3.1 .5)(2) g$ is a nonzerodivisor on $C$, and subtracting their lengths from both sides we get (1).

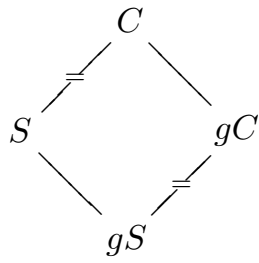

Second: Looking at the diagram of $S$-modules below, we have the two ways of inserting a module between $g C \subset C$ as on the left $g C \subset g^{\prime} C \subset C$ and on the right $g C \subset z^{e} C \subset C$. Consequently we have (i) $\ell_{S}\left(C / g^{\prime} C\right)+\ell_{S}\left(g^{\prime} C / g C\right)=$ $\ell_{S}\left(C / z^{e} C\right)+\ell_{S}\left(z^{e} C / g C\right)$ and (ii) $\ell_{S}(C / g C)=\ell_{S}\left(C / g^{\prime} C\right)+\ell_{S}\left(g^{\prime} C / g C\right)$. We get the isomorphism $C / g^{\prime} C \approx z^{e} C / g C$ because by (3.1.5)(3) $z^{e}$ is a nonzerodivisor on $C$, and subtracting their lengths from both sides of (i) we obtain (iii) $\ell_{S}\left(g^{\prime} C / g C\right)=$ $\ell_{S}\left(C / z^{e} C\right)$. Substituting (iii) in the RHS of (ii) we get (2).

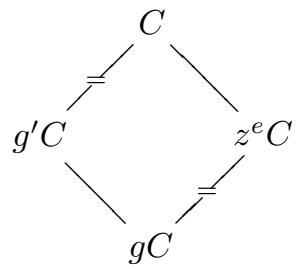


Third: Looking at the diagram of $S$-modules below, we have the two ways of inserting a module between $z S \subset C$ exhibited by the left half $z S \subset S \subset C$ and the right half $z S \subset z C \subset C$. This yields the equation $\ell_{S}(C / S)+\ell_{S}(S / z S)=$ $\ell_{S}(C / z C)+\ell_{S}(z C / z S)$. We get the isomorphism $C / S \approx z C / z S$ because by (3.1.5)(3) $z$ is a nonzerodivisor on $C$, and subtracting their lengths from both sides we get (3) because $S / z S \approx R /(F, x) R \approx W / \psi(F) W=W / M(W)^{d}$ where $\psi$ is the natural epimorphism of $R$ onto the DVR $W=R / x R$.

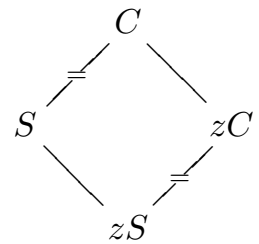

(4) follows from (3) by noting that multiplication by $z$ gives isomorphisms

$$
S / z S \approx z S / z^{2} S \approx z^{2} S / z^{3} S \approx \ldots
$$

(5) follows from (1) to (4).

Lemma 3.3. In the situation of $(3.2), \mathfrak{P}_{1}(R, F)$ is a nonempty finite set, and labelling its distinct members as $T_{1}, \ldots, T_{h}$ we have

$$
\left(R, T_{i}, F R\right)(F)=F^{\prime} \text { and }\left(R, T_{i}, G R\right)(G)=G^{\prime} \text { for } 1 \leq i \leq h
$$

and

$$
\ell_{S}\left(C / g^{\prime} C\right)=\sum_{1 \leq i \leq h} \chi\left(R, T_{i}\right) \iota\left(F^{\prime}, G^{\prime} ; T_{i}\right),
$$

where $\iota\left(F^{\prime}, G^{\prime} ; T_{i}\right)$ equals a positive integer or zero according as $T_{i}$ belongs to $\mathfrak{P}_{1}(R, G)$ or not.

Proof. Clearly $\mathfrak{P}_{1}(R, F)$ is a nonempty finite set, and labelling its distinct members as $T_{1}, \ldots, T_{h}$ we have $\left(R, T_{i}, F R\right)(F)=F^{\prime}$ and $\left(R, T_{i}, G R\right)(G)=G^{\prime}$ for $1 \leq i \leq h$. Also it is clear that $\iota\left(F^{\prime}, G^{\prime} ; T_{i}\right)$ equals a positive integer or zero according as $T_{i}$ belongs to $\mathfrak{P}_{1}(R, G)$ or not. Let $N_{i}=\phi\left(M\left(T_{i}\right) \cap B\right)$. Then clearly $N_{1}, \ldots, N_{h}$ are exactly all the maximal ideals in $C$. Let $\phi_{i}: C \rightarrow C_{i}$ be the canonical homomorphism where $C_{i}$ is the local ring $C_{N_{i}}$ (see page 144 of [Ab8]), and let us regard $C_{i}$ as an $S$-module via $\phi_{i}$. Then for $1 \leq i \leq h$ we clearly have

$$
\chi\left(R, T_{i}\right)=\chi\left(\phi_{i}(S), C_{i}\right) \text { and } \iota\left(F^{\prime}, G^{\prime} ; T_{i}\right)=\ell_{C_{i}}\left(C_{i} / \phi_{i}\left(g^{\prime}\right)\right) .
$$

Therefore we are reduced to the following:

Lemma 3.4. Let $S$ be a one dimensional local ring. Let $C$ be an overring of $S$ such that $C$ is a finite $S$-module. Let $N_{1}, \ldots, N_{h}$ be the maximal ideals in $C$. Let $\phi_{i}: C \rightarrow C_{i}$ be the canonical homomorphism where $C_{i}$ is the local ring $C_{N_{i}}$, and let us regard $C_{i}$ as an $S$-module via $\phi_{i}$. Let $g \in S$ and $g^{\prime} \in C$ be such that $\left(g^{\prime} C\right) \cap S=g S$ and the ideal $g S$ is $M(S)$-primary. Then upon letting

$$
e_{i}=\ell_{C_{i}}\left(C_{i} / \phi_{i}\left(g^{\prime}\right)\right) \text { and } f_{i}=\chi\left(\phi_{i}(S), C_{i}\right) \text { for } 1 \leq i \leq h
$$

we have

$$
\ell_{S}\left(C / g^{\prime} C\right)=\sum_{1 \leq i \leq h} e_{i} f_{i}
$$


Proof. Let $\psi: C \rightarrow Q=C / g^{\prime} C$ be the residue class ring epimorphism, and let $P=\psi(S)$. Then $P$ is a zero dimensional local ring, and $Q$ is an overring of $P$ such that $Q$ is a finite $P$-module. Let $M_{i}=\psi\left(N_{i}\right)$. Then $M_{1}, \ldots, M_{h}$ are exactly all the distinct maximal ideals in $Q$, and upon letting $\psi_{i}: Q \rightarrow Q_{i}=Q_{M_{i}}$ be the canonical homomorphism, we have that

$$
\ell_{C_{i}}\left(C_{i} / \phi_{i}\left(g^{\prime}\right)\right)=\ell_{Q_{i}} Q_{i} \text { and } \chi\left(\phi_{i}(S), C_{i}\right)=\chi\left(\psi_{i}(P), Q_{i}\right) .
$$

Therefore we are reduced to the following:

Lemma 3.5. Let $P$ be a zero dimensional local ring. Let $Q$ be an overring of $P$ such that $Q$ is a finite $P$-module. Let $M_{1}, \ldots, M_{h}$ be the maximal ideals in $Q$. Let $\psi_{i}: Q \rightarrow Q_{i}$ be the canonical homomorphism where $Q_{i}$ is the local ring $Q_{M_{i}}$, and let us regard $Q_{i}$ as an $P$-module via $\psi_{i}$. Then we have

$$
\ell_{P} Q=\sum_{1 \leq i \leq h} \ell_{P} Q_{i} \quad \text { and } \quad \ell_{P} Q_{i}=\left(\ell_{Q_{i}} Q_{i}\right) \chi\left(\psi_{i}(P), Q_{i}\right) \text { for } 1 \leq i \leq h .
$$

Proof. The first equality follows from the fact that the $P$-module $Q$ is naturally isomorphic to the direct $\operatorname{sum} Q_{1} \oplus \cdots \oplus Q_{h}$. The proof of the second equality follows from: ,

Lemma 3.6. Let $S$ and $T$ be local rings such that $T$ dominates $S$, and $T$ is residually finite algebraic over $S$. Let $N$ be a T-module of finite T-length. Then $\ell_{S} N=\left(\ell_{T} N\right) \chi(S, T)$.

Proof. We can take a sequence $0=N_{0} \subset N_{1} \subset \cdots \subset N_{l}=N$ of $T$-modules such that $\ell_{T}\left(N_{i} / N_{i-1}\right)=1$ for $1 \leq i \leq l=\ell_{T} N$. Let $\sigma_{i}: N_{i} \rightarrow N_{i} / N_{i-1}$ be the residue class $T$-epimorphism. Take $1_{i} \in N_{i} \backslash N_{i-1}$ and find a unique $T$-isomorphism $\tau_{i}: T / M(T) \rightarrow N_{i} / N_{i-1}$ such that $\tau_{i}\left(H_{T}(1)\right)=\sigma_{i}\left(1_{i}\right)$. It only remains to note that clearly $\ell_{S}(T / M(T))=\chi(S, T)$.

Proof of Theorem (3.1). In the special case when the residue field $R / M(R)$ is infinite, Theorem (3.1) follows from Lemma (3.2)(5) and Lemma (3.3) by induction on the height of $\mathfrak{P}(R, F) \cap \mathfrak{P}(R, G)$, by which we mean the smallest positive integer $h$ such that $(\mathfrak{P}(R, F) \cap \mathfrak{P}(R, G)) \cap Q_{h}(R)=\emptyset$.

The general case can be reduced to the special case by using the generic incarnation $Q^{t}(R)$ and by noting that clearly:

(1) The residue field $R^{t} / M\left(R^{t}\right)$ is infinite.

(2) The ideal $(F, G) R^{t}$ is $M\left(R^{t}\right)$-primary.

(3) $\iota\left(F, G ; R^{t}\right)=\iota(F, G ; R)$.

(4) $\mathfrak{P}\left(R^{T}, F\right) \cap \mathfrak{P}\left(R^{t}, G\right)=\left\{T^{t}: T \in \mathfrak{P}(R, F) \cap \mathfrak{P}(R, G)\right\}$.

(5) $\chi\left(R^{t}, T^{t}\right)=\chi(R, T)$ for all $T \in Q(R)$.

(6) $\operatorname{ord}_{T^{t}}\left(R^{t}, T^{t}\right)\left(F T^{t}\right)=\operatorname{ord}_{T}(R, T)(F T)$ for all $T \in Q(R)$.

(7) $\operatorname{ord}_{T^{t}}\left(R^{t}, T^{t}\right)\left(G T^{t}\right)=\operatorname{ord}_{T}(R, T)(G T)$ for all $T \in Q(R)$.

\section{Contact numbers}

In this section, in addition to the terminology of Ab11] to Ab14, we shall also use the terminology of AA1. The following theorem, (4.1), about $M(R)$-primary complete ideals in a two dimensional regular local domain $R$ with quotient field $L$ is proved in Theorem (4.6) of $\mathrm{AH} 3$. 
Theorem on Commutativity of Contact Numbers (4.1). Let

$$
I=\prod_{1 \leq i \leq h} \zeta_{R}\left(V_{i}\right)^{n(i)}
$$

where $h, n(1), \ldots, n(h)$ are positive integers and $V_{1}, \ldots, V_{h}$ are pairwise distinct members of $D(R)^{\Delta}$. Let

$$
I^{*}=\prod_{1 \leq i \leq h^{*}} \zeta_{R}\left(V_{i}^{*}\right)^{n^{*}(i)}
$$

where $h^{*}, n^{*}(1), \ldots, n^{*}\left(h^{*}\right)$ are positive integers and $V_{1}^{*}, \ldots, V_{h^{*}}^{*}$ are pairwise distinct members of $D(R)^{\Delta}$. Let $F, G, F^{*}, G^{*}$ be nonzero elements of $M(R)$ such that

$$
((F, G) R)^{-R}=I \text { and }\left(\left(F^{*}, G^{*}\right) R\right)^{-R}=I^{*} .
$$

Then we have the following.

(4.1.1) Upon letting

$$
\Phi=F+t G \text { and } \Phi^{*}=F^{*}+t G^{*},
$$

and assuming $(\bullet)\left(\Phi, \Phi^{*}\right) R^{t}$ to be $M\left(R^{t}\right)$-primary, we have

$$
c\left(R, I, I^{*}\right)=\iota\left(\Phi, \Phi^{*} ; R^{t}\right)=c\left(R, I^{*}, I\right) .
$$

(4.1.2) Assuming $I$ and $I^{*}$ have 2-generated reductions, i.e., assuming there exist elements $F, G, F^{*}, G^{*}$ satisfying $(\dagger)$, we have

$$
c\left(R, I, I^{*}\right)=c\left(R, I^{*}, I\right) .
$$

(4.1.3) Upon letting

$$
\Phi=F+t G \text { and } \Phi^{*}=F^{*}+t^{*} G^{*}
$$

we have

$$
c\left(R, I, I^{*}\right)=\iota\left(\Phi, \Phi^{*} ; R^{t, t^{*}}\right)=c\left(R, I^{*}, I\right) .
$$

We shall now show that (4.1.2) remains valid without assuming $I$ and $I^{*}$ to have 2-generated reductions.

Proposition on Commutativity of Contact Numbers (4.2). For any $M(R)$ primary complete ideals $I$ and $I^{*}$ in $R$ we have

$$
c\left(R, I, I^{*}\right)=c\left(R, I^{*}, I\right) .
$$

Proof. By the Zariski Factorization Theorem, stated as (2.4) in Ab12, we can write

$$
I=\prod_{1 \leq i \leq h} \zeta_{R}\left(V_{i}\right)^{n(i)}
$$

where $h, n(1), \ldots, n(h)$ are positive integers and $V_{1}, \ldots, V_{h}$ are pairwise distinct members of $D(R)^{\Delta}$, and

$$
I^{*}=\prod_{1 \leq i \leq h^{*}} \zeta_{R}\left(V_{i}^{*}\right)^{n^{*}(i)}
$$

where $h^{*}, n^{*}(1), \ldots, n^{*}\left(h^{*}\right)$ are positive integers and $V_{1}^{*}, \ldots, V_{h^{*}}^{*}$ are pairwise distinct members of $D(R)^{\Delta}$. Considering the two dimensional regular local domain

$$
\bar{R}=R^{t, t^{*}}
$$

and letting

$$
\bar{V}_{i}=\left(V_{i}\right)^{t, t^{*}} \text { with } \bar{I}=I \bar{R} \text { and } \bar{V}_{j}^{*}=\left(V_{j}^{*}\right)^{t, t^{*}} \text { with } \bar{I}^{*}=I^{*} \bar{R},
$$


we easily see that $\bar{V}_{1}, \ldots, \bar{V}_{h}$ are pairwise distinct prime divisors of $\bar{R}, \bar{I}$ is an $M(\bar{R})$-primary complete ideal in $\bar{R}$ with

$$
\bar{I}=\prod_{1 \leq i \leq h} \zeta_{\bar{R}}\left(\bar{V}_{i}\right)^{n(i)}
$$

$\bar{V}_{1}^{*}, \ldots, \bar{V}_{h^{*}}^{*}$ are pairwise distinct prime divisors of $\bar{R}$, and $\bar{I}^{*}$ is an $M(\bar{R})$-primary complete ideal in $\bar{R}$ with

$$
\bar{I}^{*}=\prod_{1 \leq j \leq h^{*}} \zeta_{\bar{R}}\left(\bar{V}_{j}^{*}\right)^{n^{*}(j)} .
$$

For $1 \leq i \leq h$ and $1 \leq j \leq h^{*}$ we have

$$
\operatorname{ord}_{\bar{V}_{i}} \zeta_{\bar{R}}\left(\bar{V}_{j}^{*}\right)=\operatorname{ord}_{V_{i}} \zeta_{R}\left(V_{j}^{*}\right) \text { with } \chi\left(\bar{R}, \bar{V}_{i}\right)=\chi\left(R, V_{i}\right)
$$

and

$$
\operatorname{ord}_{\bar{V}_{j}^{*}} \zeta_{\bar{R}}\left(\bar{V}_{j}^{*}\right)=\operatorname{ord}_{V_{j}^{*}} \zeta_{R}\left(V_{i}\right) \text { with } \chi\left(\bar{R}, \bar{V}_{j}^{*}\right)=\chi\left(R, V_{j}^{*}\right) .
$$

It follows that

$$
c\left(\bar{R}, \bar{I}, \bar{I}^{*}\right)=c\left(R, I, I^{*}\right) \text { and } c\left(\bar{R}, \bar{I}^{*}, \bar{I}\right)=c\left(R, I^{*}, I\right) .
$$

Now the residue field $\bar{R} / M(\bar{R})$ is obviously infinite, and hence by AH1 we know that the ideals $\bar{I}$ and $\bar{I}^{*}$ have 2-generated reductions. Hence by applying (4.1.2) to $\bar{I}, \bar{I}^{*}$, by $(\sharp)$ we conclude that $c\left(R, I, I^{*}\right)=c\left(R, I^{*}, I\right)$.

Theorem 4.3. For $V \in D(R)^{\Delta}$ let $S=\left(S_{j}, I_{j}\right)_{0 \leq j \leq \mu}$ and $S^{\prime}=\left(S_{j}^{\prime}, I_{j}^{\prime}\right)_{0 \leq j \leq \mu}$ be curvette stems belonging to $\sigma^{*}(R, V)$ for which we have $S_{\mu} \neq S_{\mu}^{\prime}$ and $\theta(R, S)=$ $\theta\left(R, S^{\prime}\right)$. For $0 \leq j \leq \mu$ let $d_{j}=\operatorname{ord}_{S_{j}} I_{j}$ and $d_{j}^{\prime}=\operatorname{ord}_{S_{j}^{\prime}} I_{j}^{\prime}$. In Note (3.9) of [AA1] we have proved that then $I_{0}+I_{0}^{\prime}$ is $M(R)$-primary and

$$
d_{j}=d_{j}^{\prime} \text { for } 0 \leq j \leq \mu-1
$$

and

(2) $\quad\left(I_{0}+I_{0}^{\prime}\right)^{-R}=\zeta_{R}(V)^{\theta(R, S)}$ with $\theta(R, S)=d_{\mu-1}$ and $\mathfrak{D}\left(R, I_{0}+I_{0}^{\prime}\right)=\{V\}$.

Now we further claim that

$$
\chi\left(S_{j}, V\right) V\left(M\left(S_{j}\right)\right)=d_{j} \text { for } 0 \leq j \leq \mu-1
$$

and

$$
\sum_{0 \leq j \leq \mu-1} \chi\left(R, S_{j}\right) d_{j}^{2}=\iota\left(I_{0}, I_{0}^{\prime} ; R\right)=\theta(R, S)^{2} \chi(R, V) c(R, V, V) .
$$

Proof of (3). Given any nonnegative integer $l \leq \mu-1$ we want to show that

$$
\chi\left(S_{l}, V\right) V\left(M\left(S_{l}\right)\right)=d_{l} .
$$

Let $\bar{R}=S_{l}$ and $\bar{\mu}=\mu-l$. Let $\bar{S}=\left(\bar{S}_{j}, \bar{I}_{j}\right)_{0 \leq j \leq \bar{\mu}}$ and $\bar{S}^{\prime}=\left(\bar{S}_{j}^{\prime}, \bar{I}_{j}^{\prime}\right)_{0 \leq j \leq \bar{\mu}}$, where for $0 \leq j \leq \bar{\mu}$ we have put $\left(\bar{S}_{j}, \bar{I}_{j}\right)=\left(S_{l+j}, I_{l+j}\right)$ and $\left(\bar{S}_{j}^{\prime}, \bar{I}_{j}^{\prime}\right)=\left(S_{l+j}^{\prime}, I_{l+j}^{\prime}\right)$. Then clearly $\bar{S}$ and $\bar{S}^{\prime}$ are members of $\sigma^{*}(\bar{R}, V)$ with

$$
\bar{S}_{\bar{\mu}}=S_{\mu} \neq S_{\mu}^{\prime}=\bar{S}_{\bar{\mu}}^{\prime} \text { and } \theta(R, S)=\theta(\bar{R}, \bar{S})=\theta\left(\bar{R}, \bar{S}^{\prime}\right)=\theta\left(R, S^{\prime}\right) .
$$


Therefore

$$
\left\{\begin{array}{l}
\text { upon letting } I=\left(I_{l}+I_{l}^{\prime}\right)^{-\bar{R}} \text { by }(2) \text { we see that } \\
I \text { is a complete } M(\bar{R}) \text {-primary ideal in } \bar{R} \text { with } I=\zeta_{\bar{R}}(V)^{\theta(R, S)} \\
\text { and by (2) we see that } \theta(R, S) \operatorname{ord}_{\bar{R}} \zeta_{\bar{R}}(V)=\operatorname{ord}_{\bar{R}} I=d_{l} .
\end{array}\right.
$$

Moreover

$$
\left\{\begin{array}{l}
\text { upon letting } I^{*}=M(\bar{R}) \text { and } V^{*}=o(\bar{R}) \text { we obviously see that } \\
I^{*} \text { is a complete } M(\bar{R}) \text {-primary ideal in } \bar{R} \text { with } I^{*}=\zeta_{\bar{R}}\left(V^{*}\right)=M(\bar{R}) .
\end{array}\right.
$$

Consequently by the definition of contact number we get

$$
c\left(\bar{R}, I, I^{*}\right)=\chi(\bar{R}, V) \operatorname{ord}_{V} \zeta_{\bar{R}}\left(V^{*}\right)=\chi\left(S_{l}, V\right) V\left(M\left(S_{l}\right)\right)
$$

and

$$
c\left(\bar{R}, I^{*}, I\right)=\theta(R, S) \operatorname{ord}_{V^{*}} \zeta_{\bar{R}}(V)=d_{l} .
$$

But by (4.2) we have $c\left(\bar{R}, I, I^{*}\right)=c\left(\bar{R}, I^{*}, I\right)$, and hence we get $\chi\left(S_{l}, V\right) V\left(M\left(S_{l}\right)\right)=$ $d_{l}$.

Proof of (4). The first equality of (4) follows from (1) and Theorem (3.1). To prove the second equality of (4), upon letting

$$
I=I^{*}=\left(I_{0}+I_{0}^{\prime}\right)^{-R} \text { with } \Phi=I_{0}+t I_{0}^{\prime} \text { and } \Phi^{*}=I_{0}+t^{*} I_{0}^{\prime}
$$

by $(2),(4.1 .3)$, and the definition of contact number, we get

$$
\iota\left(\Phi, \Phi^{*} ; R^{t, t^{*}}\right)=c(R, I, I)=\theta(R, S)^{2} \chi(R, V) c(R, V, V) .
$$

It only remains to note that, in view of (1), by (3.1) we have

$$
\iota\left(\Phi, \Phi^{*} ; R^{t, t^{*}}\right)=\iota\left(I_{0}, I_{0}^{\prime} ; R\right) .
$$

Theorem 4.4. For $V \neq V^{*}$ in $D(R)^{\Delta}$, let $S=\left(S_{j}, I_{j}\right)_{0 \leq j \leq \mu}$ and $S^{\prime}=\left(S_{j}^{\prime}, I_{j}^{\prime}\right)_{0 \leq j \leq \mu}$ be members of $\sigma^{*}(R, V)$, and let $S^{*}=\left(S_{j}^{*}, I_{j}^{*}\right)_{0 \leq j \leq \mu}$ and $S^{\prime *}=\left(S_{j}^{\prime *}, I_{j}^{\prime *}\right)_{0 \leq j \leq \mu^{*}}$ be members of $\sigma^{*}\left(R, V^{*}\right)$. Let $d_{j}=\operatorname{ord}_{S_{j}} I_{j}$ for $0 \leq j \leq \mu$, and let $d_{j}^{*}=\operatorname{ord}_{S_{j}^{*}} I_{j}^{*}$ for $0 \leq j \leq \mu^{*}$. Assume that

$$
\left\{S_{\mu}, S_{\mu^{*}}^{*}\right\} \cap\left(Q\left(S_{\mu}^{\prime}\right)\right) \cup\left(Q\left(S_{\mu^{*}}^{*}\right)\right)=\emptyset=\left\{S_{\mu}^{\prime}, S_{\mu^{*}}^{* *}\right\} \cap\left(Q\left(S_{\mu}\right)\right) \cup\left(Q\left(S_{\mu^{*}}^{*}\right)\right) .
$$

Let

and let

$$
\theta=\theta(R, S) \text { and } \theta^{*}=\theta\left(R, S^{*}\right)
$$

$\lambda$ be the smallest positive integer $\leq \min \left(\mu, \mu^{*}\right)$ such that $S_{\mu} \neq S_{\mu^{*}}^{*}$

(where we note that clearly $\lambda$ exists). Then we have

$$
\left(I_{0} I_{0}^{*}+I_{0}^{\prime} I_{0}^{\prime *}\right)^{-R}=\zeta_{R}(V)^{\theta} \zeta_{R}\left(V^{*}\right)^{\theta^{*}}
$$

with

$$
\mathfrak{D}\left(R, I_{0} I_{0}^{*}+I_{0}^{\prime} I_{0}^{*}\right)=\left\{V, V^{*}\right\}
$$

and

(3) $\iota\left(I_{0}, I_{0}^{*} ; R\right)=\iota\left(I_{0}, I_{0}^{* *} ; R\right)=\iota\left(I_{0}^{\prime}, I_{0}^{*} ; R\right)=\iota\left(I_{0}^{\prime}, I_{0}^{\prime *} ; R\right)=\sum_{0 \leq j \leq \lambda-1} \chi\left(R, S_{j}\right) d_{j} d_{j}^{*}$ with

$$
\chi(R, V) c\left(R, V, V^{*}\right) \theta \theta^{*}=\iota\left(I_{0}, I_{0}^{*} ; R\right)=\chi\left(R, V^{*}\right) c\left(R, V^{*}, V\right) \theta \theta^{*} .
$$


Proof. The proof of Theorem (4.4) is very similar to the proof of Theorem (4.3), the main difference being the fact that now we invoke the $h=2$ case of Theorem (3.4) of [AA1 instead of the $h=1$ case as reproduced in Note (3.9) of [AA1.

\section{Multiplicity SEQUenCES}

In this section, in addition to the terminology of Ab11] to [Ab14, we shall also use the terminology of AL2].

Let $(x, y)$ be generators of the maximal ideal $M(R)$ in a two dimensional regular local domain with quotient field $L$, and let $\kappa$ be a coefficient set of $R$. Theorem (5.1) stated below was proved in Theorem (4.9) of AL2 and was used in proving Theorems (4.3) and (4.4) of the previous section. The said Theorem (5.1) dealt with free points $S_{\mu} \in \pi^{*}(R, V)$ and $S_{\mu}^{\prime} \in \pi^{*}(R, V)$ of the prime divisor $V \in D(R)^{\Delta}$ of $R$. In Example (5.3) we shall show that the condition of freeness is necessary in Theorem (5.1). In (5.2) we shall prepare the groundwork for (5.3).

Theorem 5.1. Let there be given any $V \in D(R)^{\Delta}$ and let there be given any curvette stems $S=\left(S_{j}, I_{j}\right)_{0 \leq j \leq \mu} \in \sigma^{*}(R, V)$ and $S^{\prime}=\left(S_{j}^{\prime}, I_{j}^{\prime}\right)_{0 \leq j \leq \mu} \in \sigma^{*}(R, V)$ of $(R, V)$ for which we have $\theta(R, S)=\theta\left(R, S^{\prime}\right)$. Let $d_{j}=\operatorname{ord}_{S_{j}} I_{j}$ and $d_{j}^{\prime}=\operatorname{ord}_{S_{j}^{\prime}} I_{j}^{\prime}$. Then $d_{j}=d_{j}^{\prime}$ for $0 \leq j \leq \nu$ where $\nu=\mu-1$.

Note 5.2. Let $S=\left(S_{j}, x_{j}, y_{j}, \kappa_{j}\right)_{0 \leq j \leq \nu}$ be a QDT sequence of $(R, x, y, \kappa)$ with $\nu \in \mathbb{N}_{+}$such that for a sequence of integers $\nu=\nu_{0}>\nu_{1}>\cdots>\nu_{p}>\nu_{p+1}=0$ with $p \in \mathbb{N}_{+}$we have that: for every nonnegative even integer $i \leq p$ and every integer $j$ with $\nu_{i} \geq j>\nu_{i+1}$ we have that $j$ is an $X$-operation of $S$, and for every nonnegative odd integer $i \leq p$ and every integer $j$ with $\nu_{i} \geq j>\nu_{i+1}$ we have that $j$ is a $Y$-operation of $S$. Let $I_{\nu_{0}}=F_{\nu_{0}} S_{\nu_{0}}$ with $F_{\nu_{0}}=x_{\nu_{0}}^{A_{0}}+y_{\nu_{0}}^{B_{0}}$ where $A_{0}, B_{0}$ are any positive integers. Then we can find a nonzero nonunit principal ideal $I_{j}$ in $S_{j}$ with $\left(S_{j}, S_{j+1}\right)\left(I_{j}\right)=I_{j+1}$ in the following manner. By induction on $q=1,2, \ldots, p+1$ define $\left(A_{q}, B_{q}\right) \in\left(N_{+}\right)^{2}$ by putting

$$
\left(A_{q}, B_{q}\right)= \begin{cases}\left(A_{q-1}+\left(\nu_{q-1}-\nu_{q}\right) B_{q-1}, B_{q-1}\right) & \text { if odd } q>0 \\ \left(A_{q-1}, B_{q-1}+\left(\nu_{q-1}-\nu_{q}\right) A_{q-1}\right) & \text { if even } q>0\end{cases}
$$

and then for $0 \leq j \leq \nu$ define $\left(a_{j}, b_{j}, d_{j}\right) \in\left(N_{+}\right)^{3}$ by putting

$$
\left(a_{j}, b_{j}, d_{j}\right)= \begin{cases}\left(A_{0}, B_{0}, \min \left(A_{0}, B_{0}\right)\right) & \text { if } j=\nu \\ \left(A_{q-1}+\left(\nu_{q-1}-j\right) B_{q-1}, B_{q-1}, B_{q-1}\right) & \text { if } \nu_{q-1}>j \geq \nu_{q} \text { with odd } q>0 \\ \left(A_{q-1}, B_{q-1}+\left(\nu_{q-1}-j\right) A_{q-1}, A_{q-1}\right) & \text { if } \nu_{q-1}>j \geq \nu_{q} \text { with even } q>0\end{cases}
$$

and for $0 \leq j<\nu$ define

$$
I_{j}=F_{j} S_{j} \text { with } F_{j}=x_{j}^{a_{j}}+y_{j}^{b_{j}}
$$

and

$$
z_{j}= \begin{cases}x_{j} & \text { if } \nu_{q-1}>j \geq \nu_{q} \text { with odd } q>0 \\ y_{j} & \text { if } \nu_{q-1}>j \geq \nu_{q} \text { with even } q>0\end{cases}
$$

Now for $0 \leq j \leq \nu$ we clearly have

$$
d_{j}= \begin{cases}\left(A_{0}, B_{0}, \min \left(A_{0}, B_{0}\right)\right) & \text { if } j=\nu \\ B_{q-1}=b_{j}<a_{j}=A_{q-1}-\left(j-\nu_{q}\right) B_{q-1} & \text { if } \nu_{q-1}>j \geq \nu_{q} \text { with odd } q>0 \\ A_{q-1}=a_{j}<b_{j}=B_{q-1}-\left(j-\nu_{q}\right) A_{q-1} & \text { if } \nu_{q-1}>j \geq \nu_{q} \text { with even } q>0\end{cases}
$$


and hence for $0 \leq j<\nu$ we have

$$
\operatorname{ord}_{S_{j}} I_{j}=d_{j} \text { with } M\left(S_{j}\right) S_{j+1}=z_{j} S_{j+1} \text { and } z_{j}^{d_{j}} F_{j+1}=F_{j} .
$$

Therefore for $0 \leq j<\nu$ we have

(1)

$$
\left(S_{j}, S_{j+1}\right)\left(I_{j}\right)=I_{j+1} \text { with }\left(S_{j}, S_{j+1}^{*}\right)\left(I_{j}\right)=S_{j+1} \text { for all } S_{j+1}^{*} \in Q_{1}\left(S_{j}\right) \backslash\left\{S_{j+1}\right\} \text {. }
$$

In view of (3.1.3), by (1) we see that for $0 \leq j<\nu$ we have

$$
I_{j+1} \cap S_{j}=I_{j}
$$

By the displayed descriptions of $\left(A_{q}, B_{q}\right)_{0 \leq q \leq p+1}$ and $\left(d_{j}\right)_{0 \leq j \leq \nu}$ we see that

$$
d_{\nu_{q}}=\alpha_{q} A_{0}+\beta_{q} B_{0} \text { with } \alpha_{q}, \beta_{q} \text { in } N_{+} \text {for } 2 \leq q \leq p+1
$$

and

$$
d_{j}=d_{\nu_{q}} \text { for } \nu_{q-1}>j \geq \nu_{q} \text { with } 2 \leq q \leq p+1 .
$$

Given any $\left(A_{0}^{\prime}, B_{0}^{\prime}\right) \in\left(N_{+}\right)^{2}$, let $\left(A_{q}^{\prime}, B_{q}^{\prime}\right)_{1 \leq q \leq p+1}$ and $\left(a_{j}^{\prime}, b_{j}^{\prime}, d_{j}^{\prime}, I_{j}^{\prime}, F_{j}^{\prime}\right)_{0 \leq j \leq \nu}$ be as defined above with $\left(A_{0}^{\prime}, B_{0}^{\prime}\right)$ replacing $\left(A_{0}, B_{0}\right)$. By $(3)$ and (4) we get the following.

$$
\left\{\begin{array}{l}
\text { Assume that } A_{0} \leq A_{0}^{\prime} \text { and } B_{0} \leq B_{0}^{\prime} . \text { Then } d_{j} \leq d_{j}^{\prime} \text { for } 0 \leq j \leq \nu . \\
\text { Moreover, if }\left(A_{0}, B_{0}\right) \neq\left(A_{0}^{\prime}, B_{0}^{\prime}\right) \text {, then } d_{j}<d_{j}^{\prime} \text { for } 0 \leq j<\nu-1 .
\end{array}\right.
$$

Example 5.3. To show that Theorem (5.1) is not valid for nonfree points, let $S=\left(S_{j}, x_{j}, y_{j}, \kappa_{j}\right)_{0 \leq j \leq \mu}$ be a QDT sequence of $(R, x, y, \kappa)$ with $\mu \in \mathbb{N}_{+}$such that every positive integer $j \leq \mu$ is a nontranslation of $S$. Swapping $x$ and $y$ if necessary, we can arrange matters so that $\mu$ is an $X$-operation of $S$. Let $V=o\left(S_{\nu}\right)$ where $\nu=\mu-1$, and assume that $S_{\mu}$ is a nonfree point of $(R, V)$. Let $\nu_{-1}=\mu$. By Lemmas (4.4) and (4.5) of AL2 we see that there is a sequence of integers $\nu=\nu_{0}>\nu_{1}>\cdots>\nu_{p}>\nu_{p+1}=0$ with $p \in \mathbb{N}_{+}$such that for every nonnegative even integer $i \leq p$ and every integer $j$ with $\nu_{i} \geq j>\nu_{i+1}$ we have that $j$ is an $X$-operation of $S$, and for every nonnegative odd integer $i \leq p$ and every integer $j$ with $\nu_{i} \geq j>\nu_{i+1}$ we have that $j$ is a $Y$-operation of $S$. Given any positive integers $n<n^{\prime}$, let $I_{\mu}=F_{\mu} S_{\mu}$ with $F_{\mu}=x_{\mu}^{n}+y_{\mu}$ and $I_{\mu}^{\prime}=F_{\mu}^{\prime} S_{\mu}$ with $F_{\mu}^{\prime}=x_{\mu}^{n^{\prime}}+y_{\mu}$. In (5.2) take $\left(A_{0}, B_{0}\right)=(n+1,1)$ and $\left(A_{0}^{\prime}, B_{0}^{\prime}\right)=\left(n^{\prime}+1,1\right)$. This produces nonzero nonunit ideals $I_{j}$ and $I_{j}^{\prime}$ in $S_{j}$ for $0 \leq j \leq \nu$ such that

$$
\left\{\begin{array}{l}
\text { for } 0 \leq j \leq \nu \text { we have } \\
\left(S_{j}, S_{j+1}\right)\left(I_{j}\right)=I_{j+1} \text { with }\left(S_{j}, S_{j+1}^{*}\right)\left(I_{j}\right)=S_{j+1} \text { for all } S_{j+1}^{*} \in Q_{1}\left(S_{j}\right) \backslash\left\{S_{j+1}\right\} \\
\text { and } \\
\left(S_{j}, S_{j+1}\right)\left(I_{j}^{\prime}\right)=I_{j+1}^{\prime} \text { with }\left(S_{j}, S_{j+1}^{*}\right)\left(I_{j}^{\prime}\right)=S_{j+1} \text { for all } S_{j+1}^{*} \in Q_{1}\left(S_{j}\right) \backslash\left\{S_{j+1}\right\}
\end{array}\right.
$$

and

$$
\left\{\begin{array}{l}
\text { for } 0 \leq j \leq \nu \text { we have } I_{j+1} \cap S_{j}=I_{j} \text { and } I_{j+1}^{\prime} \cap S_{j}=I_{j}^{\prime} \\
\text { for } \nu_{1} \leq j \leq \mu \text { we have } \operatorname{ord}_{S_{j}} I_{j}=1=\operatorname{ord}_{S_{j}} I_{j}^{\prime} \\
\text { for } 0 \leq j<\nu_{1} \text { we have } \operatorname{ord}_{S_{j}} I_{j}<\operatorname{ord}_{S_{j}} I_{j}^{\prime}
\end{array}\right.
$$




\section{REFERENCES}

[Ab1] Shreeram Abhyankar, On the valuations centered in a local domain, Amer. J. Math. 78 (1956), 321-348. MR0082477 (18,556b)

[Ab2] Shreeram Abhyankar, Ramification theoretic methods in algebraic geometry, Annals of Mathematics Studies, no. 43, Princeton University Press, Princeton, N.J., 1959. MR.0105416 (21 \#4158)

[Ab3] S. S. Abhyankar, Resolution of singularities of embedded algebraic surfaces, 2nd ed., Springer Monographs in Mathematics, Springer-Verlag, Berlin, 1998. MR.1617523 (99c:14021)

[Ab4] Shreeram S. Abhyankar, Historical ramblings in algebraic geometry and related algebra, Amer. Math. Monthly 83 (1976), no. 6, 409-448. MR0401754 (53 \#5581)

[Ab5] Shreeram S. Abhyankar, Desingularization of plane curves, Singularities, Part 1 (Arcata, Calif., 1981), Proc. Sympos. Pure Math., vol. 40, Amer. Math. Soc., Providence, RI, 1983, pp. 1-45. MR713043 (85d:14024)

[Ab6] Shreeram S. Abhyankar, Algebraic geometry for scientists and engineers, Mathematical Surveys and Monographs, vol. 35, American Mathematical Society, Providence, RI, 1990. MR.1075991 (92a:14001)

[Ab7] Shreeram S. Abhyankar, Polynomial expansion, Proc. Amer. Math. Soc. 126 (1998), no. 6, 1583-1596, DOI 10.1090/S0002-9939-98-04183-5. MR1443142 (98g:12003)

[Ab8] S. S. Abhyankar, Lectures on Algebra I, World Scientific, 2006. MR.2253320 (2007k:13001)

[Ab9] Shreeram S. Abhyankar, Inversion and invariance of characteristic terms: Part I, The legacy of Alladi Ramakrishnan in the mathematical sciences, Springer, New York, 2010, pp. 93-168, DOI 10.1007/978-1-4419-6263-8_8. MR2744259(2012b:14057)

[Ab10] Shreeram S. Abhyankar, Dicritical divisors and Jacobian problem, Indian J. Pure Appl. Math. 41 (2010), no. 1, 77-97, DOI 10.1007/s13226-010-0017-x. MR2650101 (2011d:14107)

[Ab11] Shreeram S. Abhyankar, Pillars and towers of quadratic transformations, Proc. Amer. Math. Soc. 139 (2011), no. 9, 3067-3082, DOI 10.1090/S0002-9939-2011-10731-7. MR.2811263 (2012f:14003)

[Ab12] Shreeram S. Abhyankar, More about dicriticals, Proc. Amer. Math. Soc. 139 (2011), no. 9, 3083-3097, DOI 10.1090/S0002-9939-2011-10732-9. MR2811264(2012d:13047)

[Ab13] Shreeram S. Abhyankar, Quadratic transforms inside their generic incarnations, Proc. Amer. Math. Soc. 140 (2012), no. 12, 4111-4126, DOI 10.1090/S0002-9939-2012-11583-7. MR 2957201

[Ab14] S. S. Abhyankar, Dicritical divisors and Dedekind's Gauss lemma, Proc. Amer. Math. Soc., to appear.

[AA1] S. S. Abhyankar and E. Artal Bartolo, Algebraic theory of curvettes and dicriticals, Proc. Amer. Math., to appear.

[AH1] Shreeram S. Abhyankar and William J. Heinzer, Existence of dicritical divisors, Amer. J. Math. 134 (2012), no. 1, 171-192, DOI 10.1353/ajm.2012.0002. MR.2876143

[AH2] Shreeram S. Abhyankar and William J. Heinzer, Existence of dicritical divisors revisited, Proc. Indian Acad. Sci. Math. Sci. 121 (2011), no. 3, 267-290, DOI 10.1007/s12044-0110035-6. MR2867982(2012h:14148)

[AH3] S. S. Abhyankar and W. J. Heinzer, Rees Valuations, Proc. Amer. Math. Soc., to appear.

[AL1] Shreeram S. Abhyankar and Ignacio Luengo, Algebraic theory of dicritical divisors, Amer. J. Math. 133 (2011), no. 6, 1713-1732, DOI 10.1353/ajm.2011.0045. MR2863374

[AL2] S. S. Abhyankar and I. Luengo, Spiders and dicriticals, Proc. Amer. Math. Soc., to appear.

[Nag] Masayoshi Nagata, Local rings, Interscience Tracts in Pure and Applied Mathematics, No. 13, Interscience Publishers, a division of John Wiley \& Sons, New York-London, 1962. MR.0155856 (27 \#5790)

[Zar] O. Zariski and P. Samuel, Commutative Algebra, Springer-Verlag, 1975. MR.0384768 $(52: 5641)$

Department of Mathematics, Purdue University, West Lafayette, Indiana 47907

E-mail address: ram@cs.purdue.edu 